\title{
A Study of Prescriptive Analysis Framework for Human Care Services Based On CKAN Cloud
}

\author{
Jangwon Gim $\mathbb{D},{ }^{1}$ Sukhoon Lee $\mathbb{D}^{1},{ }^{1}$ and Wonkyun Joo $\mathbb{D}^{2}$ \\ ${ }^{1}$ Department of Software Convergence Engineering, Kunsan National University, Gunsan-si, Republic of Korea \\ ${ }^{2}$ Biomedical HPC Technology Research Center, KISTI, Daejeon, Republic of Korea \\ Correspondence should be addressed to Wonkyun Joo; joo@kisti.re.kr
}

Received 24 November 2017; Accepted 29 January 2018; Published 21 March 2018

Academic Editor: Ka L. Man

Copyright (C) 2018 Jangwon Gim et al. This is an open access article distributed under the Creative Commons Attribution License, which permits unrestricted use, distribution, and reproduction in any medium, provided the original work is properly cited.

\begin{abstract}
A number of sensor devices are widely distributed and used today owing to the accelerated development of IoT technology. In particular, this technological advancement has allowed users to carry IoT devices with more convenience and efficiency. Based on the IoT sensor data, studies are being actively carried out to recognize the current situation or to analyze and predict future events. However, research for existing smart healthcare services is focused on analyzing users' behavior from single sensor data and is also focused on analyzing and diagnosing the current situation of the users. Therefore, a method for effectively managing and integrating a large amount of IoT sensor data has become necessary, and a framework considering data interoperability has become necessary. In addition, an analysis framework is needed not only to provide the analysis of the users' environment and situation from the integrated data, but also to provide guide information to predict future events and to take appropriate action by users. In this paper, we propose a prescriptive analysis framework using a 5W1H method based on CKAN cloud. Through the CKAN cloud environment, IoT sensor data stored in individual CKANs can be integrated based on common concepts. As a result, it is possible to generate an integrated knowledge graph considering interoperability of data, and the underlying data is used as the base data for prescriptive analysis. In addition, the proposed prescriptive analysis framework can diagnose the situation of the users through analysis of user environment information and supports users' decision making by recommending the possible behavior according to the coming situation of the users. We have verified the applicability of the $5 \mathrm{~W} 1 \mathrm{H}$ prescriptive analysis framework based on the use case of collecting and analyzing data obtained from various IoT sensors.
\end{abstract}

\section{Introduction}

A number of sensor devices are widely distributed and used today owing to the accelerated development of IoT technology. In particular, this technological advancement has allowed users to carry IoT devices with more convenience, wear them for longer periods of time with greater comfort, and are evolving to become capable of collecting accurate user biometrics. As a result, there have been numerous studies in this area, such as those extracting the lifelog of users based on data collection from their various IoT devices and those detecting unusual signals from user biometrics. Among these, studies on smart healthcare services have been particularly active.

There are studies which conduct real-time monitoring and collection of user biometrics using wearable devices such as Fitbits and smartwatches to diagnose user behavior. Apple provides its "cloud" service in order to collect and use personal biosignal information communicated from an Apple watch. Fitbit, which contains a three-axis accelerometer, GPS, and other various sensors, is another device which also collects data and provides open API $[1,2]$. Hence, users may utilize various devices based on their personal preference, despite all devices measuring the same bioinformation such as heartbeat, acceleration, and blood flow. It takes the advantage of choosing a suitable device according to the context and preference of a particular user; however, a solution is required to ensure data interoperability in integrating identical biometrics or data generated from different devices. Currently, studies are looking into user behavior analysis and prediction based on user biometrics generated based on the geographic information or atmospheric environment of a 
specific area. The investigation aims at extracting the characteristics of users in the same circumstance and their unique patterns and also at analyzing their biometrics in order to provide users with customized healthcare services. Data for this investigation must not be device-dependent and should be collected and integrated according to each type of biometrics in order to provide corresponding smart healthcare services to specific user groups or individuals displaying similar patterns. Therefore, an integrated platform is required to collect, process, and manage data compatible with the cloud environment.

There are also studies aiming to analyze the behavioral patterns of specific users or groups of users based on user lifelog data and to predict behaviors based on the analysis. In particular, a previous study extracted elderly people's walking speed patterns and collected unusual signs such as reduced speed and frequent falls to predict incidents of falling or tripping. In other words, this study applied the data analysis methodology and attempted not only to collect past and present lifelog data, but also to develop techniques to predict possible future incidents. However, although this prediction technique warns users of eminent signs and potential future events based on their lifelog data, it does not provide information necessary for more effective decision making such as how to avoid such risks or events. As such, it is important to study methods that provide prescriptive analysis in preparation for specific signs and events.

Data analysis from a business aspect can be categorized into the following three categories [3]:

(i) Descriptive analytics: a set of technologies and processes to understand and analyze business outcomes using data.

(ii) Predictive analytics: prediction of results based on data input using a range of statistical and mathematical techniques. This method analyzes a variety of relationships based on predictions and suggests a model maximize business outcomes.

(iii) Prescriptive analytics: a set of mathematical techniques to determine complex targets, requirements, and limitations to improve business outcomes. This approach determines various alternatives and guides based on results drawn from descriptive analytics and predictive analytics.

Prescriptive analytics was launched as one of the hype cycle's emerging technologies announced by the Gartner Group in 2013 and has since received increasingly growing attention in business areas $[4,5]$. Prescriptive analysis, as one type of data analysis technique, provides predictions and context-customized information. This technique is used to support more effective decision making based on various ideas when business decision makers, such as CTOs and CEOs, analyze and predict complex situations.

A previous study investigated big data analysis for healthcare in the big data environment [6]. Such analysis can provide smart healthcare services through event detecting mechanisms other than the existing data analysis methods. In other words, techniques for obtaining accurate healthcare data analysis in the big data environment, which allows the acquisition of a colossal amount of diverse data, play an instrumental role in providing smart human care services. Big data analysis is performed to support decision making based on insights into the user context and the planning, management, and learning for systematic data use. There are descriptive, predictive, and prescriptive analysis models used for data analysis. Each analysis model has been separately used according to existing services and purposes. However, it is necessary to merge them to draw final analytical results in the big data and cloud computing environments in which diverse data are integrated. Therefore, it is necessary to perform technological analysis on the users and their context to accurately diagnose their current status and also to integrate and analyze collected wearable sensor data and cognitive context information. In addition, various guides should be provided to users in urban environments where complicated and diverse events take place, in order to provide optimal smart healthcare services. Guides are well known to broaden the range of choices available for users in decision making. A prescriptive analysis technique should be introduced here to provide such guides in accordance with the user context [7]. To this end, this study proposes a $5 \mathrm{~W} 1 \mathrm{H}$-based prescriptive analysis method based on the prescriptive analysis methodology to provide user-customized smart healthcare services and a data integration architecture featuring interoperability with data in cloud computing environments. The $5 \mathrm{~W} 1 \mathrm{H}$ approach can help to analyze contexts and situations of users because the $5 \mathrm{~W} 1 \mathrm{H}$ can seek to answer six basic questions such as "what," "when," "where," "who," "why," and "how" in gathering information about a plain. Therefore, nowadays, the $5 \mathrm{~W} 1 \mathrm{H}$ is being studied in the field of event processing to extract events from big data as a template or as a single ontology schema, to recognize the situation from events, and to detect abnormalities from events [8-12]. As one of the smart human care services, we apply the $5 \mathrm{~W} 1 \mathrm{H}$ approach to provide recommendation results for actions to be taken by users in specific situations.

Recently, there has been a pursuit of studies to reveal and increase the reusability of government-produced data, given the growing importance of data sharing in governmental and public organizations. This facilitated quality management of metadata and raw data, and several data portal systems supporting catalog management, and metadata interoperability were developed as a result. A representative example is the CKAN data portal. The CKAN is an open source data portal platform and is one of the tools used to build open data websites (https://ckan.org). The CKAN helps to manage data collection and sharing and is used by national and regional governments and various research institutions to collect a greater amount of data. In the CKAN system, users can save and share metadata schema to explain data catalogs and data resources saved on the system and as well as search for data. Diverse CKAN Extensions are being developed, and the system is applied in numerous fields for integrated management of open data. A typical example is the sharing of data produced in smart 
cities. Maps and geographical information produced in smart cities can be saved and shared in the CKAN. Files of various types (XML, CSV, JSON, or SHP files) can be used in a RESTful way, and it is possible to connect the RDF store to save the ontology. The CKAN shares information on education, transportation infrastructures, energy consumption, local climate, and environment as an open data portal, thus allowing devices or persons to access various forms of data on services required by citizens or other forms of data. Furthermore, catalog and metadata saved on the CKAN can be harvested based on the OAI-PMH (Open Archives Initiative Protocol for Metadata Harvesting), allowing physical metadata repositories set up in other localities or separate CKANs used in other domains to be interconnected. In essence, it allows a cloud computing environment to be established. This indicates that it is possible to use the network as an interoperable base system for datasets defined by the different schemas. A previous study was conducted to save and analyze, in real time, data related to the economy, crime, and safety generated in smart cities based on the benefits of the CKAN including its functional extensibility and data interoperability $[13,14]$. As a result of such efforts, there have been various systems capable of data integration and analysis using the CKAN. These features can also be applied in the integration of wearable sensor data. Data interoperability can be ensured by exchanging and harvesting metadata and schema required for the integration of raw data produced by wearable sensors, and sensor data can be incorporated based on integrated and interlinked schematic information.

The remainder of this study is structured as follows: Section 2 introduces related studies. Section 3 presents a smart healthcare framework for prescriptive analysis services. Section 4 explains user scenarios with sample data for explaining prescriptive analytics services based on $5 \mathrm{~W} 1 \mathrm{H}$. Section 5 discusses the proposed framework. Section 6 presents conclusions and the future study directions.

\section{Related Works}

2.1. Sensor Information and Data Integration. A sensor is a module which observes stimuli and measures their value. This is called stimulus-sensor-observation (SSO) pattern, and such patterns are suitable for expressing and defining sensor information [15]. Semantic sensor network (SSN) ontology is an ontology developed by W3C based on SSO patterns [16]. Sensor information can be expressed in the existing sensor network environment as consisting of sensor nodes and sink nodes. In particular, W3C recently developed SOSA (sensor, observation, sample, and actuator) ontology to facilitate applications in the IoT environment. This is an extended version of SSN ontology and is capable of defining additional information such as sample, actuator, and provenance [17]. Sensor registry system (SRS) is a system for registering and managing sensor-related information [18]. The principal focus of this system is on metadata, and sensor information is registered in the heterogeneous ubiquitous sensor network environment. However, the SRS simply consists of metadata such as sensor location, sensor type, model name, and access type but does not collect the actual values registered by the sensor. SRS merely provides metadata from sensors to facilitate the integration of significant sensor information from specific sensor models. With this method, SRS is able to cover all sensors which are unable to transmit sensor data to cloud storage through the Internet. On the other hand, path prediction-based sensor registry system (PP-SRS) focuses on providing real-time metadata using mobile devices by applying path prediction technology to the SRS [19]. The existing SRS is able to provide sensor information as a location-based service as it manages sensor information including location coordinates. The PP-SRS provides sensor information by learning user paths through mobile devices such as users' smartphones and predicting the movement paths of users accordingly. This renders the PP-SRS more immediate and seamless.

2.2. Healthcare Platform and Analysis Method. Efforts to apply cloud computing to the healthcare field have been ongoing for several years now [20]. Doukas et al. attempted to access various healthcare information of patients with mobile devices through a cloud [21], and Rolim et al. suggested sensors attached to medical equipment in hospitals to save data in real time into a cloud service [22]. On the other hand, Zhang et al. proposed a healthcare cyberphysical system, aimed to primarily integrate medical data on a cloud environment-obtained from hospitals, the public health sector, research institutions, drug manufacturers, financial institutions, and wearable device manufacturers-and to analyze big data [23]. However, there are various issues in data sharing on cloud services [24]. In particular, patient information always bears security and privacy risks. Patient information saved on in-hospital systems such as medical devices or EMR contains protected health information. For this reason, the United States, for instance, enacted the HIPAA legislation and imposed strong regulations to control the storage of sensitive information on external servers. Contrarily, wide efforts are dedicated to converting patient healthcare data into a cloud service based on the recent advancements of digital healthcare technology, deidentification, and anonymization technology $[25,26]$. In addition, the reliability of biosignal data collected from wearable devices has noticeably increased owing to the recent evolution of wearable devices such as smartwatches [27]. Diverse platforms have been developed to collect data, and it is now possible to measure patients' status and information on their surrounding environments using sensors through IoT and wearable devices [28]. Products such as S-Health by Samsung [29], Google Fit by Google [30], HealthKit by Apple [31], and Fitbit by Fitbit Inc. [32] all use their respective platforms to save data collected from smartwatches and various IoT devices onto a cloud server. Most of these platforms use RESTful API to provide users with collected data, and users can easily obtain their data through simple identification process and a URL. Owing to the evolution of wearable devices, there is a growing application of this technology to medical and healthcare studies using data obtained through wearable devices in place of medical devices [33]. Sensor technology coupled with wearable devices is already producing significant findings from studies, 
and users can be provided with diverse healthcare services by integrating clinical data obtained from sensors on a cloud. In order to achieve this, a cloud-based framework is required for medical information integration. In addition, a prescriptive analytics service should be in place for accurate analysis and prediction of user context in order to provide custom information and to support users' decision making.

In relation to analytic services, several studies have been launched in order to provide context-customized services by analyzing data generated from humans to support human care services. For example, there has been research conducted on tools, technology, and methodology for healthcare data value analysis [34]. The prescriptive analysis technique in the medical support area allows the provision of more varied information when used for treating patients than for analyzing users' current contexts and is used to benchmark the resulting rise in hospital revenue [35]. In other words, various guides have been suggested to support family doctors to treat patients, providing predicted outcomes according to the particular treatment and thus allowing doctors to choose the most suitable guide. As a result, hospitals are able to analyze outcomes based on the chosen guide. This prescriptive analysis technique is also used in business situations to compare performance outcomes. Thus, it is necessary to apply the prescriptive analysis technique to users and patients. Prescriptive results produced through prescriptive analysis can be interlinked with healthcare services. Further studies are required on prescriptive analysis techniques which can provide more appropriate results through analysis and prediction of user context information.

\section{Architecture}

This chapter explores data sharing platforms for providing prescriptive analysis-based human care services.

It proposes the implementation and design of cloudbased data analysis service and elaborates on a set of processes used for human care services. The major principle of the proposed architecture design is to support appropriate decision making in decision-requiring situations such as disasters, activities, and events based on user context information. The proposed system includes the $5 \mathrm{~W} 1 \mathrm{H}$ (what, when, where, who, why, and how) categorization process to perform a prescriptive analysis. The proposed architecture diagnoses the current contexts of users based on diagnostic data saved on the CKAN-based cloud computing environment and also predicts eminent situations, events, and incidents. It provides users with prescriptive guides on when and how they must respond to a specific event based on predicted results. Figure 1 presents the system architecture for data integration using a cloud environment and for providing prescriptive analysis services. Therefore, the architecture is divided into two layers. The first is the cloud computing layer, and the second is the layer for data analysis. The cloud computing layer is shown at the bottom of Figure 1. The cloud computing environment is indispensable regarding the sharing of a large amount of big data. Hence, the cloud computing environment enables the integration of data and the sharing of analytical tools. Also, open data used in different application domains (environment, economy, and disasters) can be interlinked using the CKAN to establish a cloud computing environment. The data obtained from wearable sensors are saved on a repository where sensor schema is defined. Context information in a cloud computing environment is established through wearable sensors (temperature, humidity, carbon dioxide, and heartbeat) and various open data. Here, the reliability and accuracy of wearable sensor data must be guaranteed for more accurate context analysis, and the transparency must also be guaranteed in data integration. Upon the diversification of wearable sensor types, however, problems may arise in data integration when the data storing schema do not correspond even though the sensor data are measured in an identical environment as the different types of products used for measurement or when no common data are in place that can interlink separate datasets. Therefore, catalogs should be shared which contain standardized metadata for data integration. The catalogs and their properties and types are shared on the CKAN data portal. The CKAN data portal and repositories in each domain can mutually harvest and share information based on the OAI-PMH (Open Archives Initiative Protocol for Metadata Harvesting). This extends CKAN harvester to parse OAI-PMH metadata sources and import datasets. It supports metadata schemas such as DC (Dublin Core) and CMDI (Component MetaData Infrastructure). Through the OAI-PMH protocol, CKANs in the cloud can share their catalogs and datasets, and the metadata and properties included in each catalog are integrated and extended based on the existing registered common concepts in the cloud. Therefore, common concepts are continually extended by newly added metadata and attributes. As a result, it is used as a knowledge graph for inference of implicit situation information, derivation of relationships between sensor data, and analysis of sensor data. Therefore, the context information of each user collected from the wearable sensors/devices is transmitted to each CKAN server, and each transmitted sensor data is integrated and stored in CKAN cloud. When the data is integrated into the CKAN cloud, catalogs, ontologies, and common concepts can be applied to integrate each sensor data from different sensors. The second data analysis layer is shown at the top of Figure 1. The data analysis layer consists of three components as shown in Figure $1(2-1,2-2,2-3)$. The first is to extract events from the aggregated data obtained from the cloud computing layer, where the events are classified as $5 \mathrm{~W} 1 \mathrm{H}$. We use three analytical techniques: descriptive analytics, predictive analytics, and prescriptive analytics (2-1). In the 2-1 step, events are extracted based on $5 \mathrm{~W} 1 \mathrm{H}$ factors with rules and conditions which can explain users' situations and contexts. At the first step of 2-1, descriptive analytics method extracts basic statistics information from the knowledge base stored in the CKAN cloud. After that, based on the results of 2-1 phase, future events coming up to the user (e.g., increase heartbeat, lower body temperature, and a surge of carbon dioxide) are extracted (2-2). The following stage predicts trends using machine learning algorithm based on the extracted context information. Potential variables, conditions, and limitations in specific contexts are 


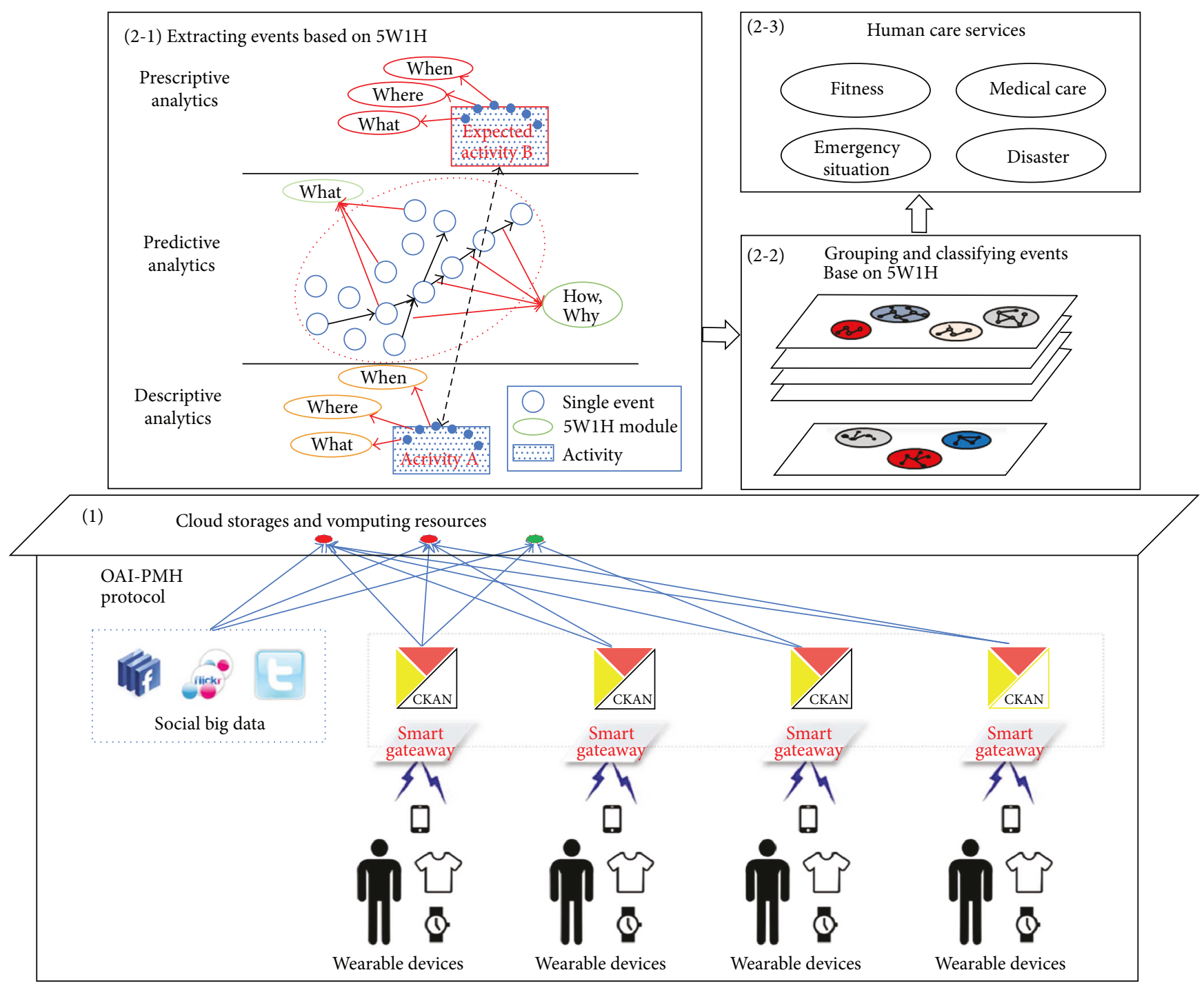

FIGURE 1: Overall architecture based on CKAN.

selected either through supervised learning or through unsupervised learning. In the $2-3$ phase, the diagnosed context and predicted outcomes obtained from these results are used to provide guides which help users to make decisions, and this process is defined as prescriptive analysis. Prescriptive information can be obtained through this prescriptive analysis, which was not possible through diagnosis and predictions. Users can make prompt and accurate decisions from a group of available response options. In this way, smart human care services can be provided to users who may have to deal with big data and various other situations.

\section{Service Scenario}

This chapter explains how datasets are collected and the implementation of prescriptive analysis with wearable devices using the CKAN through an overall scenario. The CKAN-based data integration method and the prescriptive analysis process are presented through a specific case. The case describes how firefighters collect integrated data through turnout gears and available devices and how a human care service is provided based on this mechanism.

4.1. Overall Scenario. Figure 2 shows the flow and structure of the service scenario. This scenario starts from the wearable devices of the user. The user is wearing a smartphone, smartwatch, and smart clothes. In the CKAN-based data integration area, (1) data are extracted from the user's wearable devices, and the data from the wearable devices are integrated using ontology such as SSN ontology. (2) Extracted sensor values are processed into datasets as a unified schema for the same sensor information by matching this information with SSN ontology. (3) The datasets are stored in the CKAN repository. On the other hand, prescriptive analysis (PA) should first be conducted in order to provide human care services as suggested by this paper. For this, (4) the dataset to be defined and its corresponding ontology is to be searched. Searched datasets are, respectively, processed through descriptive analytics, predictive analytics, and prescriptive analytics. (5) Scripts resulting from these analyses are stored 


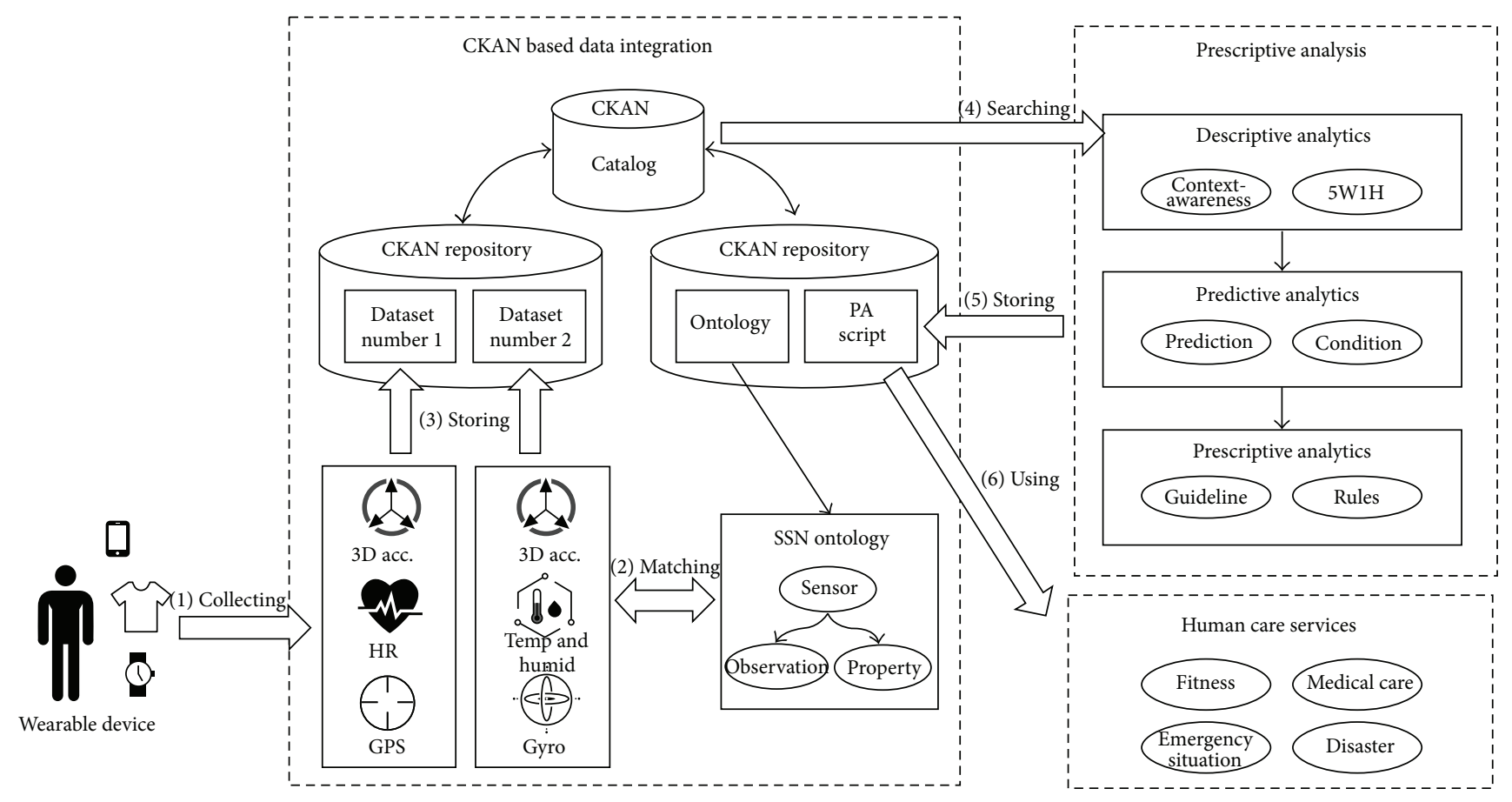

Figure 2: A conceptual model for scenario.

TABLE 1: Collected sensor types of smart garments.

\begin{tabular}{|c|c|c|c|c|}
\hline Sensor & Measurement type & Type name & Datatype & Unit of measure \\
\hline External temperature sensor & Temperature & ex_temp & Double & Celsius, Fahrenheit \\
\hline Heat flux sensor & Temperature & hf_temp & Double & Celsius, Fahrenheit \\
\hline \multirow{3}{*}{ GPS } & Latitude & lat & Double & \multirow{3}{*}{$\mathrm{E}, \mathrm{W}, \mathrm{S}, \mathrm{N}$} \\
\hline & Longitude & lon & Double & \\
\hline & Altitude & alt & Double & \\
\hline \multirow{3}{*}{$3 \mathrm{D}$ accelerometer } & $x$-axis acceleration & acc_x & Double & \multirow{3}{*}{$\mathrm{mV} / \mathrm{g}$} \\
\hline & $y$-axis acceleration & acc_y & Double & \\
\hline & $z$-axis acceleration & acc_z & Double & \\
\hline \multirow{2}{*}{ Gas sensor } & CO concentration & co & Double & \multirow{2}{*}{$\mathrm{ppm}$} \\
\hline & $\mathrm{CO}_{2}$ concentration & $\mathrm{co} 2$ & Double & \\
\hline HR sensor & Heart rate & $\mathrm{hr}$ & Integer & bpm \\
\hline $\mathrm{SPO}_{2}$ sensor & Arterial oxygen saturation & spo2 & Integer & Percentage \\
\hline
\end{tabular}

again on the CKAN repository as PA scripts. (6) Diverse human care services are provided using PA scripts afterward.

4.2. CKAN-Based Data Integration. For a more expansive scenario, it must first be assumed that the firefighters use wearable devices including sensors attached to their turnout gear and a smartwatch. The ProeTEX project, for instance, developed smart garments such as turnout gear, $t$-shirt, and a pair of boots with 12 sensors, 2 alarms, and 4 network modules [36]. Table 1 provides the definition of different sensor types used in this scenario out of the group of sensors attached to smart garments. Based on the sensors, we extracted features such as type, type name, datatype, and unit of measure. The features can be important elements to integrate sensor data considering their semantics from diverse
IoT sensors when the data derived from IoT sensors is integrated based on common concepts such as an ontology schema stored in the CKAN cloud.

Figure 3 demonstrates how the external temperature sensor is associated to SSN ontology among the sensors on the smart garments. The sensor, measurement type, and type name columns in Table 1 are mapped into "sosa: Sensor," "sosa: ObservableProperty," and "sosa: Observation," respectively. Sensor observations generated from a single user can be bound through "sosa: FeatureofInterest" like the case of "ProeTEX/user1." Various wearable device sensors and lifelogs can be integrated at the SSN ontology schema level through ontology matching. RDF generated as a result of matching is stored on the CKAN repository with corresponding datasets. In fact, SOSA is capable of a raw data 


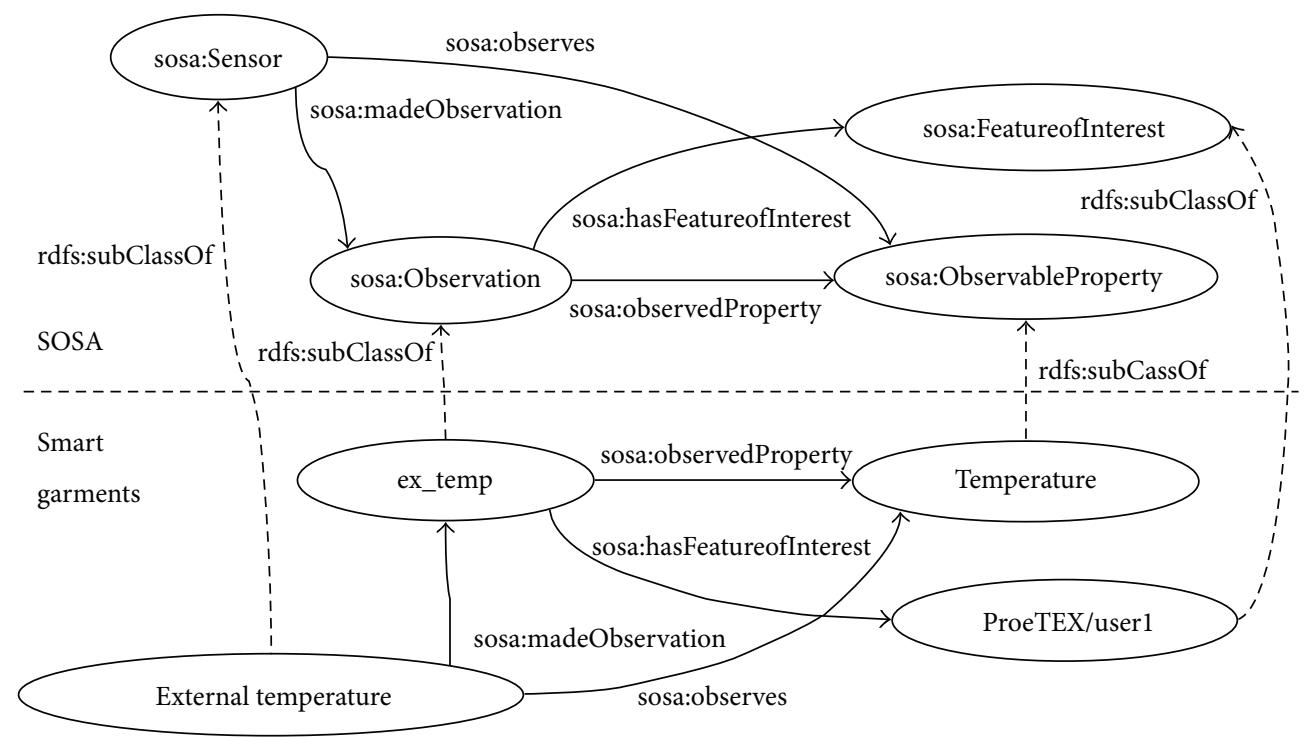

FIgURE 3: A sensor ontology to merge ontology instances.

TABLE 2: Features classified as $5 \mathrm{~W} 1 \mathrm{H}$.

\begin{tabular}{lcc}
\hline Features & Measurement type & 5W1H \\
\hline ex_temp & Temperature & What \\
hf_temp & Temperature & What \\
lat, lon, alt & Latitude, longitude, altitude & Where \\
acc_x, acc_y, acc_z & $x$-, $y$-, $z$-axis acceleration & What \\
co & CO concentration & What \\
co2 & CO2 concentration & What \\
hr & Heart rate & What \\
spo2 & Arterial oxygen saturation & What \\
o2 & Oxygen ratio of firefighter respirator & What \\
user_id & User identifier & Who \\
time & Timestamp & When \\
date & Timestamp & When \\
\hline
\end{tabular}

(observation value) level expression, but a schema level integration was considered in this scenario.

4.3. 5W1H-Based Prescriptive Analysis. The prescriptive analysis method suggested in this paper begins by searching the data stored on the CKAN. As shown in Figure 2, descriptive analytics, predictive analytics, and prescriptive analytics are consecutively conducted in a prescriptive analysis. Here, data required for descriptive analytics are categorized into $5 \mathrm{~W} 1 \mathrm{H}$ using context-awareness technology. Table 2 shows how the features of each sensor are classified into $5 \mathrm{~W} 1 \mathrm{H}$ through descriptive analytics.

The $5 \mathrm{~W} 1 \mathrm{H}$ classification is performed depending on the "sosa:observableProperty" of each measurement type during the ontology matching described in Section 4.2. In the step of a predictive analysis, each feature can be extracted and classified through machine learning algorithms and data mining methods considering the $5 \mathrm{~W} 1 \mathrm{H}$. First, data collected from the sensors are learned, and a concrete phenomenon is predicted. Prediction results and conditions are categorized into factors and defined based on the predicted situation. For example, a phenomenon is predicted using many features such as "the external temperature will rise by about 10 degrees in 10 minutes." Then, features such as "in 10 minutes" and "the external temperature will rise by about 10 degrees" and their predicted values are extracted. Moreover, situations caused by specific conditions can be predicted as well. It is possible to suggest a prediction result such as "it is dangerous if the external temperature is higher than 200 degrees Celsius." Through this process, conditions like "the external temperature is higher than 200 degrees Celsius" can be extracted. Table 3 shows results of a concrete situation and extractable conditions through predictive analytics.

In summary, prescriptive analytics suggests a guideline for specific situations to users. Users are vulnerable to various risks when they do not have any previous experience or are unfamiliar with the situation because they are unsure on what to do. The application of the previously defined predictive situation and limitation will allow suggesting a systematically more immediate and effective guideline to users. Table 4 presents guidelines and rules drawn from the combinations of predictions and conditions.

Prescriptive analysis is highly effective, particularly in critical firefighting situations. The example scenario suggests a set of guidelines to help firefighters predict and avoid danger when they are in, or are highly likely to be in, a dangerous situation. No matter how experienced a particular firefighter may be, these kinds of systematic context-awareness guidelines are highly useful when they find themselves in extreme fire incidents [37].

\section{Discussion}

Contexts should be accurately diagnosed and analyzed to provide prescriptive analysis services for diverse situations 
TABLE 3: Predictions and conditions extracted from predictive situations and limitations using 5W1H factors.

\begin{tabular}{|c|c|}
\hline re situations (PS) & tions (L), and $5 \mathrm{~W} 1 \mathrm{H}$ features \\
\hline $\begin{array}{l}\text { PS1: the external temperat } \\
5 \mathrm{~W} 1 \mathrm{H} \rightarrow\{\text { What, When, } \mathrm{H}\end{array}$ & will rise by about 10 degrees in 1 \\
\hline $\begin{array}{l}\text { PS2: the heart rate will rise } \\
\text { of the respirator falls by } 10 \\
5 \mathrm{~W} 1 \mathrm{H} \rightarrow\{\text { What, When, } \mathrm{H}\end{array}$ & $40 \mathrm{bpm}$ in 10 minutes, and oxygen $\mathrm{r}$ \\
\hline $\begin{array}{l}\frac{\mathrm{CO} 2}{5 \mathrm{~W} 1} \mathrm{H} \rightarrow\{\text { concentration will ris } \\
\text { What, When, } \mathrm{H}\end{array}$ & $1000 \mathrm{ppm}$ in $\underline{5 \text { minutes. }}$ \\
\hline $\begin{array}{l}\text { It is dangerous if the exterr } \\
5 \mathrm{~W} 1 \mathrm{H} \rightarrow\{\mathrm{Wh} \text { at, How }\}\end{array}$ & emperature is higher than $200 \mathrm{deg}$ \\
\hline $\begin{array}{l}\text { It is dangerous if the oxyg } \\
5 \mathrm{~W} 1 \mathrm{H} \rightarrow\{\mathrm{Wh} \text { at, How }\}\end{array}$ & tio of the respirator is lower than 1 \\
\hline $\begin{array}{l}\text { It is dangerous if the } \underline{\mathrm{CO} 2} \\
5 \mathrm{~W} 1 \mathrm{H} \rightarrow\{\mathrm{Wh} \text { at, How }\}\end{array}$ & entration is higher than $10,000 \mathrm{pp}$ \\
\hline & \\
\hline Prescriptive rule & Guideline \\
\hline$\{\mathrm{p} 1, \mathrm{p} 2, \mathrm{p} 3, \mathrm{c} 1\} \rightarrow \mathrm{GL} 1$ & $\begin{array}{c}\text { GL1) Go outside within } 5 \text { minutes. } \\
\text { 5W1 } \mathrm{H} \rightarrow\{\text { What, When, Where }\}\end{array}$ \\
\hline$\{\mathrm{p} 1, \mathrm{p} 3, \mathrm{p} 4, \mathrm{c} 2\} \rightarrow \mathrm{GL} 2$ & $\begin{array}{l}\text { GL2) Start charging your firefighter } \\
\text { respirator within } 5 \text { minutes. } \\
5 \mathrm{~W} 1 \mathrm{H} \rightarrow\{\text { What, When, How }\}\end{array}$ \\
\hline$\{\mathrm{p} 5, \mathrm{p} 6, \mathrm{c} 3\} \rightarrow \mathrm{GL} 3 \vee \mathrm{GL} 4$ & $\begin{array}{l}\text { GL3) Put on your firefighter } \\
\text { respirator immediately. } \\
\text { 5W1H } \rightarrow \text { What, When }\} \\
\text { GL4) Go outside immediately. } \\
\text { 5W1H } \rightarrow \text { What, When }\}\end{array}$ \\
\hline
\end{tabular}

in a cloud computing environment. Data collected for this purpose should be managed transparently and quickly integrated. Semantic interoperability between wearable sensor data should also be guaranteed. To achieve this, a CKANbased cloud data portal which is able to share common schema or catalogs is proposed. In order to perform prescriptive analysis by drawing analytical results from the data portal, the following factors should be considered:

(i) The need for machine-readable raw data

(ii) The need for data interlinking, to link the meanings of data

(iii) The need for sharing metadata and catalogs used on different domains

(iv) The need for stable processing and complex event processing techniques for big sensor data

Furthermore, in order to provide various guidelines based on prescriptive analytics results excluding those from descriptive and predictive analytics, a set of rules and limitations should be established at the prescriptive analysis stage. To achieve this, simulations of diverse situations should be performed to generate multiple candidate recommendations for suggesting an optimum guide by configuring environmental variables and conditions.
With the increase of large-scale data and the innovative development of data processing technology, an environment that can handle general knowledge, as well as specific areas, is being created. For example, Graph search on Facebook has suggested a new search method that can find the most relevant "people," "photos," "places," and "interests" on Facebook. This shows that semantic retrieval is possible over existing syntax search through graphical knowledge structure. Google's knowledge base also builds a database of vast amounts of knowledge and common sense and uses it for a variety of intelligent services. Therefore, this knowledge base can be usefully used in the field of smart human care service. Based on the knowledge base, we can derive various smart services considering user environment or find additional semantic information which cannot be derived from existing single or partial sensor data by using various sensor data. For this purpose, the proposed CKAN cloud environment that can share common concept as one of knowledge base and can collect and store big data will be useful. Specific situations can be simulated through an accumulation of scenarios, rules, and limitations for each situation, and this increases the accuracy of prescriptive analysis services. Interlinking information from many different domains saved on the cloud generates situation perceiving knowledge graphs, which allows the derivation of new rules from a set of rules and limitations. In this manner, the range of guidelines provided by prescriptive analysis services can continue to expand, and it will be possible not only to provide information on specific singular situations but also to suggest guidelines for possible situations in complex events and areas of interest. Hence, the $5 \mathrm{~W} 1 \mathrm{H}$ method enables efficient extraction of events that are meaningful to the user from a vast amount of events. In other words, when a dangerous disaster situation or difficult decision-making situation is encountered from the viewpoint of a specific user, an action guide can be provided to the user through an indicator analysis. In addition, when real-time collection and processing is required, a template of a formalized form is required to automatically classify and extract meaningful data within a fixed time, and the proposed $5 \mathrm{~W} 1 \mathrm{H}$ method can be applied. 


\section{Conclusion}

The wearable sensor technology is advancing at a remarkable pace, along with the recent development of IoT technology. As a result, sensors have generated countless data, and current studies are investigating methods to draw meaningful analysis from big data. Various studies are currently being conducted in the human care services area to diagnose and analyze user contexts in various situations. However, such analysis services do not provide guidelines on how to respond to eminent risk factors or events. In other words, they only provide analytical results to users and do not offer any guidelines as to when a user should take action, what kind of action they should take, and how to perform such action in the event of a dangerous situation (fall, car accident, fire, and disaster). In order to analyze and diagnose given situations accurately, it is imperative to integrate data in many different forms that are publicly shared by many local and related fields and as well as the user's wearable sensor information. An analysis technique is also required to provide an appropriate prescriptive analysis result (guideline) based on integrated data. This paper proposed an architecture to perform prescriptive analysis based on CKAN, which is widely used for data sharing to solve such issues. Using a $5 \mathrm{~W} 1 \mathrm{H}$-based prescriptive analysis method presented in this study, the applicability of the architecture was verified based on a user scenario. The proposed system architecture can be widely applied in many fields for analyzing smart cities and urban environments and responding to situations. In this way, smart human care services can be provided to users wearing wearable sensors and devices.

\section{Conflicts of Interest}

The authors declare that there is no conflict of interest regarding the publication of this article.

\section{Acknowledgments}

This work was supported by the "Development of biomedical data network analysis technology for dementia researches (K-18-L03-C02)" funded by Korea Institute of Science and Technology Information. This work was supported by the National Research Foundation of Korea (NRF) grant funded by the Korea government (MSIT) (NRF-2017R1C1B2012065).

\section{References}

[1] T. Fritz, E. M. Huang, G. C. Murphy, and T. Zimmermann, "Persuasive technology in the real world: a study of longterm use of activity sensing devices for fitness," in Proceedings of the 32nd Annual ACM Conference on Human Factors in Computing Systems - CHI '14, pp. 487-496, Toronto, ON, Canada, 2014, ACM.

[2] T. Rault, A. Bouabdallah, Y. Challal, and F. Marin, "A survey of energy-efficient context recognition systems using wearable sensors for healthcare applications," Pervasive and Mobile Computing, vol. 37, pp. 23-44, 2017.
[3] S. K. Song, D. H. Jeong, J. Kim, M. Hwang, J. Gim, and H. Jung, "Research advising system based on prescriptive analytics," in Future Information Technology, pp. 569-574, Springer, Berlin, Heidelberg, 2014.

[4] A. Linden and J. Fenn, "Understanding Gartner's hype cycles," Strategic Analysis Report, R-20-1971. Gartner, Inc., Stamford, CT, USA, 2003.

[5] S. John Walker, "Big data: a revolution that will transform how we live, work, and think," International Journal of Advertising, vol. 33, no. 1, pp. 181-183, 2014.

[6] A. Belle, R. Thiagarajan, S. M. R. Soroushmehr, F. Navidi, D. A. Beard, and K. Najarian, "Big data analytics in healthcare," BioMed Research International, vol. 2015, Article ID 370194, 16 pages, 2015.

[7] W. Raghupathi and V. Raghupathi, "An overview of health analytics," Journal of Health \& Medical Informatics, vol. 4, p. 132, 2013.

[8] S. Sharma, R. Kumar, P. Bhadana, and S. Gupta, "News event extraction using $5 \mathrm{~W} 1 \mathrm{H}$ approach \& its analysis," International Journal of Scientific \& Engineering Research, vol. 4, no. 5, pp. 2064-2068, 2013.

[9] L. Xie, H. Sundaram, and M. Campbell, "Event mining in multimedia streams," Proceedings of the IEEE, vol. 96, no. 4, pp. $623-647,2008$.

[10] W. Wang, D. Zhao, L. Zou, D. Wang, and W. Zheng, "Extracting $5 \mathrm{~W} 1 \mathrm{H}$ event semantic elements from Chinese online news," in International Conference on Web-Age Information Management, pp. 644-655, Springer, Berlin, Heidelberg, 2010.

[11] W. Wang, "Chinese news event $5 \mathrm{~W} 1 \mathrm{H}$ semantic elements extraction for event ontology population," in Proceedings of the 21st International Conference Companion on World Wide Web - WWW '12 Companion, pp. 197-202, Lyon, France, 2012, ACM.

[12] W. Zhou, Y. Zhang, X. Su, Y. Li, and Z. Liu, "Semantic role labeling based event argument identification," International Journal of Database Theory and Application, vol. 9, no. 6, pp. 93-102, 2016.

[13] T. Käfer, J. Umbrich, and A. Hogan, "Polleres: Towards a Dynamic Linked Data Observatory," in Workshop on Linked Data on the Web(LDOW), vol. 937, 2012.

[14] S. Lalithsena, P. Hitzler, A. Sheth, and P. Jain, "Automatic domain identification for linked open data," in 2013 IEEE/ WIC/ACM International Joint Conferences on Web Intelligence (WI) and Intelligent Agent Technologies (IAT), vol. 1, pp. 205212, Atlanta, GA, USA, November 2013, IEEE.

[15] K. Janowicz and M. Compton, "The stimulus-sensorobservation ontology design pattern and its integration into the semantic sensor network ontology," in Proceedings of the 3rd International Conference on Semantic Sensor Networks SSN'10, vol. 668, pp. 64-78, Shanghai, China, November 2010, CEUR-WS.org.

[16] M. Compton, P. Barnaghi, L. Bermudez et al., "The SSN ontology of the W3C semantic sensor network incubator group," Web Semantics: Science, Services and Agents on the World Wide Web, vol. 17, pp. 25-32, 2012.

[17] Semantic Sensor Network Ontology, http://www.w3.org/TR/ vocab-ssn/.

[18] D. Jeong, "Framework for seamless interpretation of semantics in heterogeneous ubiquitous sensor networks," International Journal of Software Engineering and Its Applications, vol. 6, no. 3, pp. 9-16, 2012. 
[19] S. Lee, D. Jeong, D. K. Baik, and D. K. Kim, "Path prediction method for effective sensor filtering in sensor registry system," International Journal of Distributed Sensor Networks, vol. 11, no. 7, article 613473, 2015.

[20] A. M. H. Kuo, "Opportunities and challenges of cloud computing to improve health care services," Journal of Medical Internet Research, vol. 13, no. 3, article e67, 2011.

[21] C. Doukas, T. Pliakas, and I. Maglogiannis, "Mobile healthcare information management utilizing cloud computing and android OS," in 2010 Annual International Conference of the IEEE Engineering in Medicine and Biology, pp. 1037-1040, Buenos Aires, Argentina, August 2010, IEEE.

[22] C. O. Rolim, F. L. Koch, C. B. Westphall, J. Werner, A. Fracalossi, and G. S. Salvador, "A cloud computing solution for patient's data collection in health care institutions," in 2010 Second International Conference on eHealth, Telemedicine, and Social Medicine, pp. 95-99, St. Maarten, Netherlands, February 2010, IEEE.

[23] Y. Zhang, M. Qiu, C. W. Tsai, M. M. Hassan, and A. Alamri, "Health-CPS: healthcare cyber-physical system assisted by cloud and big data," IEEE Systems Journal, vol. 11, no. 1, pp. 88-95, 2017.

[24] S. P. Ahuja, S. Mani, and J. Zambrano, "A survey of the state of cloud computing in healthcare," Network and Communication Technologies, vol. 1, no. 2, 2012.

[25] L. Devadass, S. S. Sekaran, and R. Thinakaran, "Cloud computing in healthcare," International Journal of Students' Research in Technology \& Management, vol. 5, no. 1, pp. 25-31, 2017.

[26] N. Mohammed, B. C. M. Fung, P. C. K. Hung, and C.-K. Lee, "Centralized and distributed anonymization for highdimensional healthcare data," ACM Transactions on Knowledge Discovery from Data, vol. 4, no. 4, pp. 1-33, 2010.

[27] J. Bughin, M. Chui, and J. Manyika, "Clouds, big data, and smart assets: ten tech-enabled business trends to watch," McKinsey Quarterly, vol. 56, no. 1, pp. 75-86, 2010.

[28] F. De Arriba-Pérez, M. Caeiro-Rodríguez, and J. Santos-Gago, "Collection and processing of data from wrist wearable devices in heterogeneous and multiple-user scenarios," Sensors, vol. 16, no. 9, p. 1538, 2016.

[29] Samsung Health, https://health.apps.samsung.com.

[30] Google Fit, https://www.google.com/fit.

[31] Apple HealthKit, https://www.apple.com/ios/health.

[32] Fitbit, https://www.fitbit.com.

[33] B. Reeder and A. David, "Health at hand: a systematic review of smart watch uses for health and wellness," Journal of Biomedical Informatics, vol. 63, pp. 269-276, 2016.

[34] T. L. Strome and A. Liefer, Healthcare Analytics for Quality and Performance Improvement, Wiley, Hoboken, NJ, USA, 2013.

[35] D. B. Nash, "Harnessing the power of big data in healthcare," American Health \& Drug Benefits, vol. 7, no. 2, pp. 69-70, 2014.

[36] D. Curone, E. L. Secco, A. Tognetti et al., "Smart garments for emergency operators: the ProeTEX project," IEEE Transactions on Information Technology in Biomedicine, vol. 14, no. 3, pp. 694-701, 2010.

[37] S. Patel, H. Park, P. Bonato, L. Chan, and M. Rodgers, "A review of wearable sensors and systems with application in rehabilitation," Journal of NeuroEngineering and Rehabilitation, vol. 9, no. 1, p. 21, 2012. 


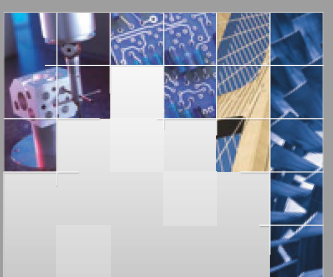

\section{Enfincering}
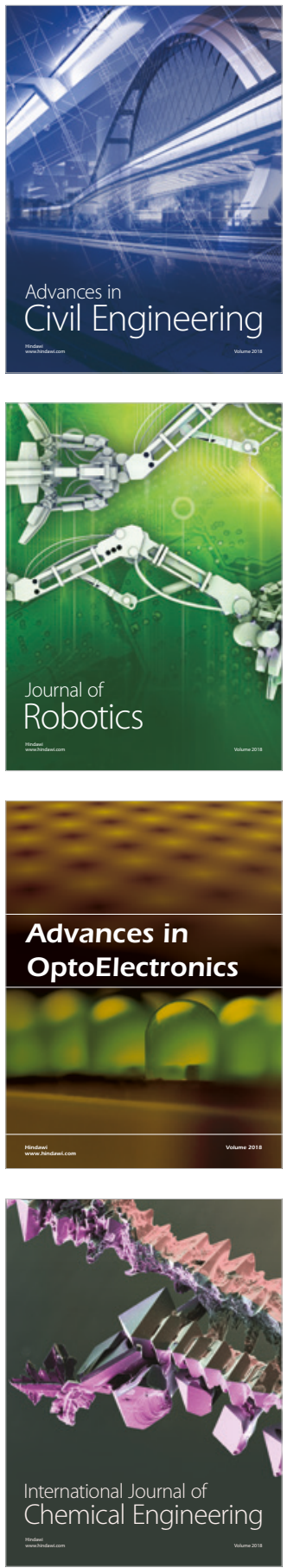

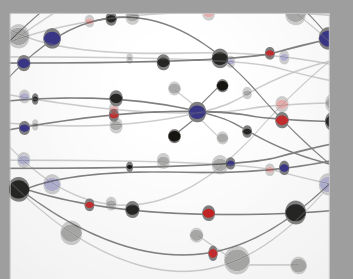

\section{Rotating \\ Machinery}

The Scientific World Journal

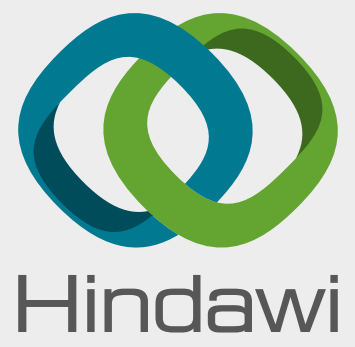

Submit your manuscripts at

www.hindawi.com
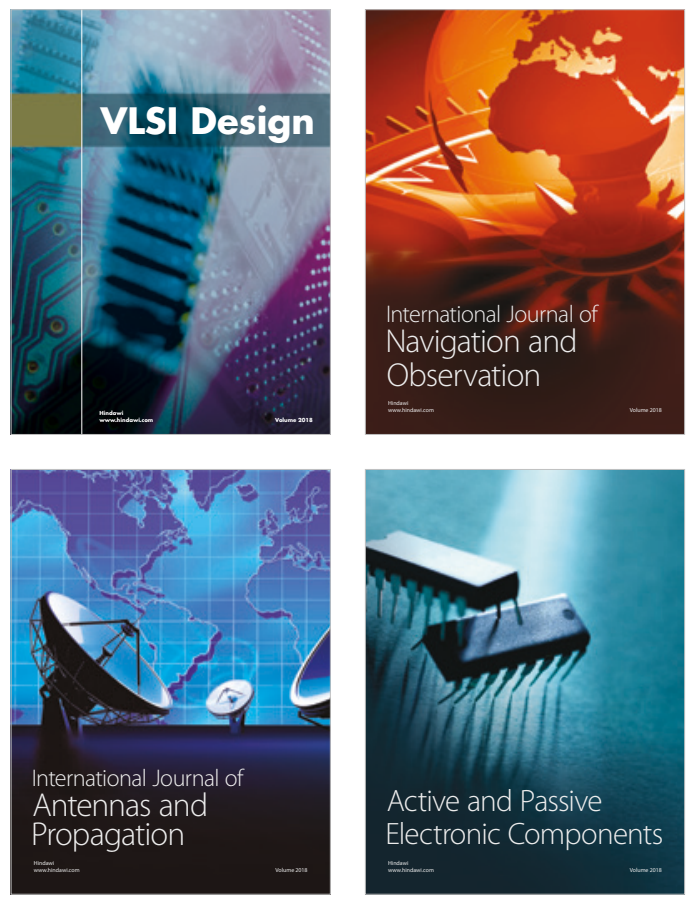
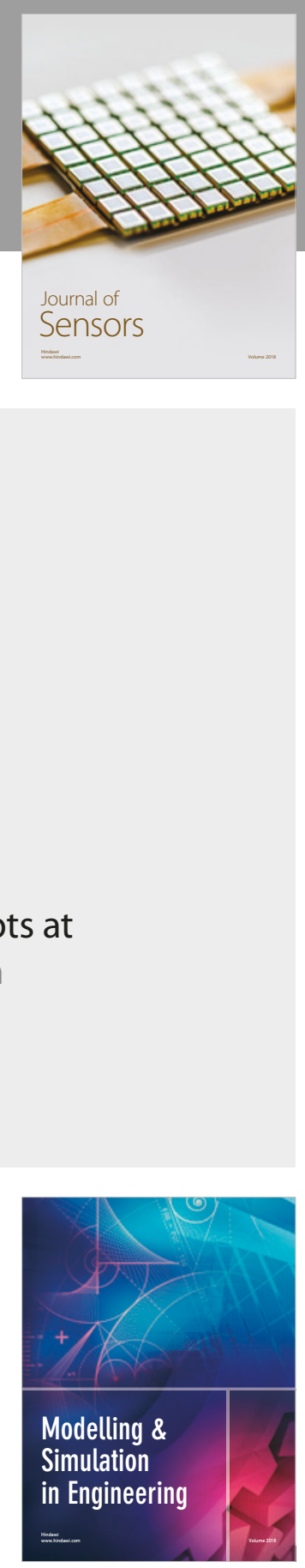

\section{Advances \\ Multimedia}
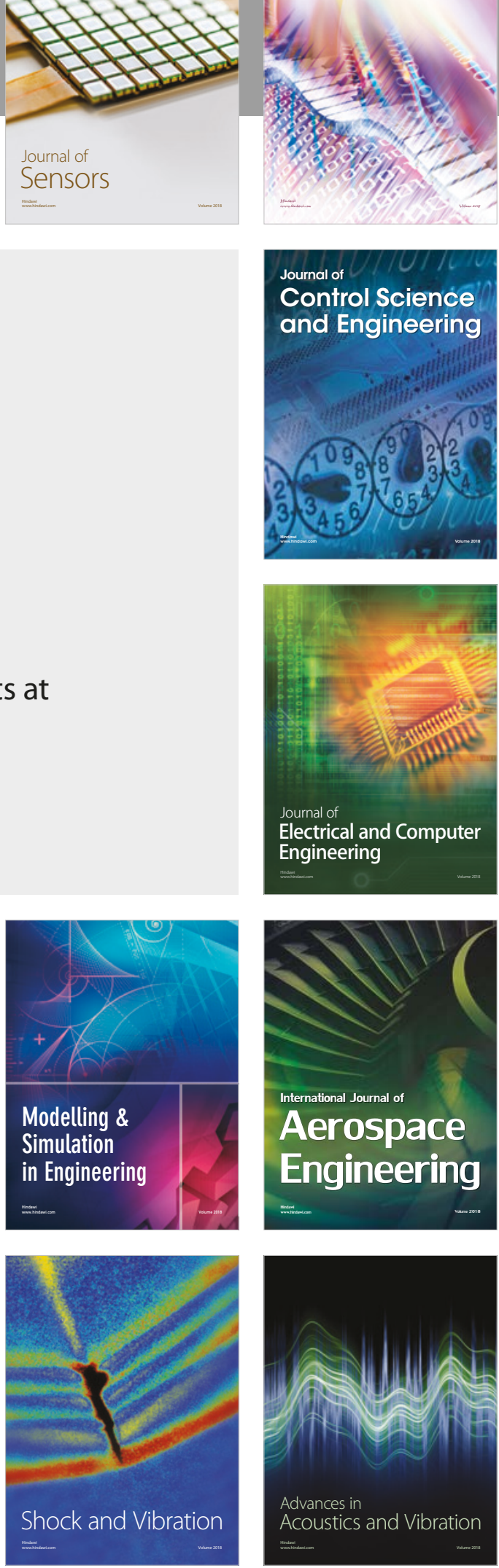